\title{
FRAME BASED RECONSTRUCTION OF SIGNALS IN MULTIRATE IMPLEMENTATION
}

\author{
KHOLE TIMOTHY POUMAI, S. K. KAUSHIK AND POONAM MANTRY ${ }^{\dagger}$
}

\author{
Date of Receiving : $\quad 01.05 .2018$ \\ Date of Revision : $\quad 06.04 .2019$ \\ Date of Acceptance : 16.04 .2019
}

\begin{abstract}
We define multidimensional discrete time Zak transform (MDTZT) and discuss various properties of MDTZT. We show that MDTZT is relevant to discrete sampling of multivariate discrete time signals. Also, we study multivariate discrete time Weyl Heisenberg (MDTWH) systems for oversampling and critical sampling schemes. By utilizing Zak transform, we give the formulations of the existence of MDTWH frames, tight MDTWH frames and dual of MDTWH frames in the context of oversampling. Moreover, we show that MDTZT provides a good method to evaluate the coefficients of the MDTWH system expansion in the case of oversampling. Also, we discuss the sampling results of the MDTWH system for critical sampling. Finally, we give the applications of MDTWH frames to reconstruction of the multivariate Weyl Heisenberg system in the periodic space $\mathrm{L}^{2}\left([0,1)^{d}\right)$.
\end{abstract}

\section{Introduction}

Let $\mathscr{H}$ be a real (or complex) separable Hilbert space with inner product $\langle.,$.$\rangle . A$ family $\left\{f_{n}\right\}_{n \in \mathbb{Z}} \subseteq \mathscr{H}$ is a frame (or Hilbert frame) for $\mathscr{H}$, if there exist constants $A, B>0$ such that

$A\|f\|^{2} \leq \sum_{n=-\infty}^{\infty}\left|\left\langle f, f_{n}\right\rangle\right|^{2} \leq B\|f\|^{2}$, for all $f \in \mathscr{H}$.

The scalars $A$ and $B$ are called the lower and upper frame bounds of the frame, respectively. They are not unique. If $A=B$, then $\left\{f_{n}\right\}$ is called an $A$-tight frame and if $A=B=1$, then $\left\{f_{n}\right\}$ is called a Parseval frame. The inequality in (1.1) is called the

2010 Mathematics Subject Classification. 42C15, 42C30, 42C05, 46B15.

Key words and phrases. Zak transform, Weyl Heisenberg frames, multidimensional sampling. The authors pay their sincere thanks to the referee for his critical remarks and suggestions which have improved the paper significantly.

Communicated by: Askari Hemmat

${ }^{\dagger}$ Corresponding author. 